\title{
Un análisis empírico sobre la capacidad de absorción tecnológica de la industria brasileña
}

\author{
Pablo Felipe Bittencourt y Ricardo Giglio
}

RESUMEN

En el presente artículo se indican y analizan evidencias empíricas de la absorción tecnológica externa propiciada por actividades que la empresa realiza internamente. Se establecieron indicadores de aprendizaje interno y externo que se aplicaron en modelos de causalidad estadística, utilizados como método para distinguir posibles formas de absorción tecnológica. Los sectores de la actividad industrial son las unidades básicas de referencia de la investigación a nivel de los tres dígitos de la Clasificación Nacional de Actividades Económicas (CNAE), que aprovecha la información emanada de la Encuesta de Innovación Tecnológica (PINTEC por sus siglas en portugués) del Brasil. Según los resultados, se advierte que las actividades de investigación y desarrollo (I+D) a nivel interno son la principal fuente de absorción tecnológica, seguidas por los conocimientos generados a partir del "aprendizaje práctico" y las "prácticas de capacitación".

PABLABRAS CLAVE

CLASIFICACIÓN JEL

AUTORES
Industria, empresas industriales, innovaciones, capacidad de absorción, tecnología, investigación y desarrollo, Brasil

$\mathrm{O} 32, \mathrm{O} 33, \mathrm{O} 38$

Pablo Felipe Bittencourt es profesor del Programa de Posgrado en Economía de la Universidad Federal de Santa Catarina. pablofelipe.bittencourt@gmail.com

Ricardo Giglio es doctorando en Economía en la Universidad de Kiel. rgiglio@gmail.com 


\section{I}

\section{Introducción}

La capacidad de desarrollar innovaciones que tengan repercusión en los mercados nacional e internacional es decisiva para reforzar la competitividad en un contexto de creciente mundialización de la competencia. Las novedades con un grado de repercusión superior usualmente demandan un conjunto notable y complejo de conocimientos, que en parte solo se encuentran fuera del límite de la empresa. Debido al aumento del volumen y la complejidad de los conocimientos potencialmente útiles, la tarea de absorberlos es cada vez menos simple. La adaptación a esa realidad suele suponer modificaciones de las rutinas para ampliar las capacitaciones internas, lo que permite incluso mejorar las condiciones para reconocer las oportunidades del ambiente externo. El proceso de aprendizaje en que las empresas participan con miras a adaptarse a esas condiciones puede entenderse como la ampliación de sus capacidades de absorción. En el artículo de Cohen y Levinthal (1990), la capacidad de absorción se define en función de la habilidad de la empresa para reconocer el valor de la información externa, asimilarla y aplicarla para fines comerciales. Básicamente, esa habilidad puede suponer conocimientos generados en los departamentos formales de I+D, en el entorno de producción de la empresa o simplemente derivados de los individuos que forman parte de ella.

Las referencias empíricas centran la investigación en la influencia de formas internas y externas de aprendizaje en la generación de innovaciones, como en los análisis de Vega-Jurado y otros (2008); Veugelers (1997); Nieto y Quevedo (2005); Tsai y Wang (2009); Jensen y otros (2007); Caloghirou, Kastelli y Tsakanikas (2004), y Cassiman y Veugelers (2006) ${ }^{1}$. Sin embargo, en estos análisis generalmente se incluyen evidencias que pueden clasificarse como "subproductos" de los resultados del enfoque principal de los trabajos y, por ello, no pueden considerarse evidencias empíricas del fenómeno eficazmente respaldadas ${ }^{2}$.

Gracias a los trabajos citados con anterioridad, se promovió recientemente una labor centrada en investigar la capacidad de absorción de las empresas. Ejemplos de ello son los análisis de Arora y Gambardella (1994); Stock, Greis y Fischer (2001); Schmidt (2005); Murovec

\footnotetext{
1 Es importante indicar que, en muchos trabajos, los resultados se basan en la propia suposición de que las actividades de I+D a nivel interno revisten relevancia para la capacidad de absorción.

2 Gran parte de los resultados recientes se fundamentan en medidas poco directas, lo que deja dudas sobre su validez.
}

y Prodan (2009), y Tsai (2009). En estos trabajos, el uso de alguna fuente externa de conocimiento se considera, normalmente, como un indicador suficiente de que la empresa posee alguna capacitación para absorberlo. A partir de ese punto, se procura avanzar en la comprensión de las actividades internas de la empresa capaces de ampliar la absorción de conocimientos externos. Según los resultados, se advierte que las actividades de I+D a nivel interno complementan un conjunto más amplio de actividades $^{3}$.

En la investigación que se desarrolla a continuación se ofrecen evidencias cuantitativas adicionales acerca del papel de las actividades internas de I+D, capacitación y "aprendizaje práctico" para la absorción de los conocimientos tecnológicos generados fuera de la empresa, capaces de producir innovaciones de producto de alto impacto. El objeto de la investigación son los patrones sectoriales de aprendizaje definidos en Bittencourt (2012). La referencia sectorial resulta conveniente porque permite diferenciar conjuntos de sectores según las características del aprendizaje. Ese enfoque es decisivo, por lo menos desde que Pavitt (1984) señaló diferencias y semejanzas entre sectores en cuanto a sus procesos sectoriales de cambio tecnológico ${ }^{4}$.

El método de relación causal estadística resultó ser adecuado, ya que permite poner a prueba la hipótesis referida al doble efecto de las actividades de I+D, la capacitación y el "aprendizaje práctico" para generar innovaciones de gran repercusión.

Además de esta Introducción y de una presentación del marco referencial teórico en la sección II, en el trabajo se incluyen otras cuatro secciones: en la tercera se presentan los patrones sectoriales de aprendizaje, en la cuarta se aborda el marco referencial metodológico, en la quinta se discuten los resultados empíricos y en la sexta se formulan las principales conclusiones.

\footnotetext{
${ }^{3}$ La característica doble o las dos fases de las inversiones en I+D se refieren a: i) la capacidad de generar nuevos conocimientos aplicables directamente a los productos y procesos que las empresas desarrollan, y ii) la ampliación de la capacidad de absorber conocimientos externos (Cohen y Levinthal, 1989). En este artículo se admite que esas mismas características pueden observarse en otras dos actividades de innovación, definidas como "aprendizaje práctico" y "aprendizaje mediante capacitación".

${ }^{4}$ Con ello no se está aceptando la clasificación de Pavitt (1984) como la mejor posible para un análisis del caso brasileño en el período en cuestión, ya que si bien la pertinencia de ese trabajo y de muchas de sus derivaciones es innegable, aquí se considera que las conclusiones se restringen a la historia y la geografía que se propuso investigar.
} 


\section{II}

\section{Marco referencial teórico y analítico}

Esta sección se subdivide en cinco subsecciones. En la primera se introducen y discuten el concepto de capacidad de absorción y algunas evidencias empíricas. En las tres siguientes se aborda el aumento de la capacidad de absorción vinculado, respectivamente, a las actividades de I+D a nivel interno, de capacitación y a aquellas derivadas del aprendizaje en las rutinas de producción, concepto que se denominó "aprendizaje práctico". Por último, en esta sección se incluye una breve discusión teórica sobre los conceptos de capacidad de absorción y absorción tecnológica, cuya pretensión es instigar al lector a realizar nuevas investigaciones teóricas.

\section{Capacidad de absorción: concepto y evidencias empíricas}

Las innovaciones son el resultado de la combinación de conocimientos nuevos. Dosi (1988) destacó esa combinación como reflejo de la búsqueda de una solución a problemas que requieren conocimientos derivados, a veces, de las experiencias pasadas $\mathrm{y}$, otras veces, de conocimientos formalizados (en especial, de aquellos generados por las ciencias naturales) $)^{5}$. Los procesos de aprendizaje que emanan de esa búsqueda se definen en función del uso de una o más fuentes de información y conocimientos, que pueden ser internas o externas con respecto a las empresas ${ }^{6}$. Debido a los costos que involucra, las empresas solo participan en procesos de búsqueda de innovaciones si perciben oportunidades de lucro todavía no exploradas?

\footnotetext{
5 Para Dosi (1988), los procesos de búsqueda y la adopción de nuevos productos y procesos se definen según la combinación compleja de diversos elementos, tales como la capacitación y el estímulo generado dentro de cada empresa y en cada industria, la situación de la ciencia en diferentes sectores, las características del patrón de consumo, el patrón de competencia de la industria, la estructura financiera, las tendencias macroeconómicas y las políticas públicas, entre otros. A pesar de que se reconoce la pertinencia de esos factores, sería imposible considerarlos todos en un análisis como el de este artículo.

${ }^{6}$ Entre las más investigadas se encuentran los proveedores, los clientes, los competidores, y las universidades y centros de investigación.

7 El concepto de innovación como proceso interactivo derivado del aprendizaje interno y externo está ampliamente aceptado. Diversas líneas de investigación se desarrollan a partir de ese concepto, aunque con enfoques sensiblemente diferentes, entre ellas los medios innovadores (Maillat, 1996) y los sistemas de innovación, ya sea en la variante nacional (Lundvall, 1992; Nelson, 1993; Freeman, 1987), sectorial (Breschi y Malerba, 1997; Malerba, 2002), regional (Cooke y Morgan, 1998) o local (Cassiolato y Lastres, 2003).
}

Sin embargo, estar preparado para percibir y aprovechar las oportunidades tecnológicas depende en cierta medida de la acumulación de conocimientos pertinentes. En los estudios se ha prestado especial atención a las actividades de I+D, desde que Cohen y Levinthal (1989) destacaron su doble efecto (dual effect) vinculado a la generación de conocimientos directamente aplicables a productos y procesos, así como al aumento de la capacidad de comprensión y absorción de conocimientos generados por potenciales socios tecnológicos externos, tales como universidades, centros de investigación, proveedores y clientes.

Desde entonces surgió un conjunto de trabajos empíricos centrados exclusivamente en esa forma de ampliar la capacidad de absorción, sin considerar apuntes de los propios autores referidos a la diversidad de las formas utilizadas para absorber conocimientos generados fuera de los límites de la empresa. Incluso en la introducción del clásico artículo de 1990 sobre capacidad de absorción, Cohen y Levinthal (1990) destacaron las características de la mano de obra empleada, las operaciones rutinarias de manufactura de la empresa y la propia experiencia derivada de las actividades de producción, como otros condicionantes de esa capacidad.

La capacidad de absorción de cada empresa se construye en un proceso que depende de decisiones pasadas (path dependence) y que puede comprender diversas etapas, lo que ciertamente requiere una labor considerable, es decir, que no es posible ampliarla solo mediante el aprendizaje práctico, destacado en Arrow (1962). Mowery, Oxley y Silverman (1996) sostienen que la capacidad de absorción consiste en un conjunto amplio de habilidades necesarias para lidiar con el componente tácito del conocimiento que se transferirá desde las fuentes externas, pero también en la habilidad para modificarlo. Nótese que la complejidad que supone la transferencia de conocimientos tácitos refuerza la idea de que la adquisición de conocimientos precisa de una labor continua para comprender los hechos pertinentes que están teniendo lugar fuera de los límites de la empresa.

Zahra y George (2002) reconocen la complejidad del concepto y avanzan teóricamente al subdividirlo en cuatro dimensiones: i) la adquisición propiamente dicha, relacionada con la capacidad para adquirir conocimientos externos críticos para las operaciones de la empresa, en 
que la intensidad, la dirección y la velocidad con que se emprenden las actividades constituyen los elementos decisivos de esa capacidad; ii) la asimilación, vinculada a las etapas de análisis, comprensión e interpretación de los conocimientos externos obtenidos; iii) la transformación, referida a la capacidad de la empresa para desarrollar y perfeccionar sus rutinas, de modo que los nuevos conocimientos se combinen eficazmente con los que ya existen, y iv) la exploración de los conocimientos, integrándolos a las rutinas estructuradas, a objeto de permitir que la empresa pueda sustentar durante largos períodos los beneficios de los nuevos conocimientos asimilados y transformados.

De modo general, el concepto de capacidad de absorción se refiere a la complementariedad ${ }^{8}$ de los conocimientos internos y externos en el proceso de innovación, que se expresa en la capacidad continua de la empresa para aprender del ambiente externo y desarrollar a partir de ahí experiencias propias que se acumulan en sus rutinas de producción e innovación. Para Mowery, Oxley y Silverman (1996), su importancia puede resumirse en la comprensión de que participar en interacciones de manera eficaz puede depender de la capacidad de absorción.

Luego, el desarrollo de habilidades en un ámbito específico colocaría a la empresa en una situación de ventaja para absorber nuevos conocimientos en ese ámbito. Tal prerrogativa puede ser decisiva en el entorno de incertidumbre característico de la competencia capitalista, pues determina la capacidad de la empresa para evaluar el potencial comercial de posibles decisiones productivas y tecnológicas.

Debido a que el concepto es amplio y complejo, resulta difícil realizar una medición cuantitativa. Tal vez ello explique el número limitado de contribuciones empíricas. Según se indicó en la Introducción, gran parte de los análisis en la materia son secundarios y otros, como el de Stock, Greis y Fischer (2001), tienen predilección por las actividades de I+D a nivel interno.

\footnotetext{
8 Véase Caloghirou, Kastelli y Tsakanikas (2004). Existen incluso resultados como los que presentan Laurensen y Salter (2006), que sugieren un efecto de sustitución entre la búsqueda de nuevos conocimientos externos y las actividades internas de I+D. Al considerar los costos relacionados con la búsqueda fuera de la empresa, se hace hincapié en que, bajo cierto nivel de dispendios, el incremento de gastos puede afectar negativamente al desempeño innovador. En ese sentido, se admiten los potenciales beneficios teóricos de la variedad de conocimientos vinculada a la diversidad de fuentes, pero se resalta que el grado de apertura debe ser ponderado por los costos conexos a dicha apertura.
}

Más recientemente, sucesivas gestiones con miras a incrementar las formas de cuantificar el fenómeno permitieron revelar importantes contribuciones a los estudios de la disciplina de economía de la innovación. Se observa que variables vinculadas a la calidad de la mano de obra, las actividades de capacitación y las actitudes emprendedoras se mostraron, a veces, tan importantes como el destacado uso de I+D a nivel interno, o incluso más relevantes (Murovec y Prodan, 2009; Schmidt, 2005; Arbussa y Coenders, 2007; Tsai, 2009).

En el Brasil, el estudio de De Negri (2006) continúa siendo la principal referencia. Sobre la base de Schmidt (2005), la autora destaca el uso de fuentes externas de información como evidencia de absorción tecnológica, investigando su vinculación a las actividades de I+D y a las características de la mano de obra empleada en las empresas brasileñas. En el análisis, las fuentes externas de información se dividen en dos categorías, a saber, las empresariales y las académicas. Los resultados se destacan por el perfil de la mano de obra y las actividades de I+D directamente relacionadas con la capacidad de absorción, y asimismo que las iniciativas de capacitación de las empresas, para ser efectivas, dependen de la capacidad de esta de retener a las personas que recibieron capacitación en ella.

\section{Capacidad de absorción y de generación de nuevos conocimientos a partir de las actividades de I+D a nivel interno}

Cohen y Levinthal (1990) ofrecen una perspectiva clave para evaluar la capacidad de absorción de las actividades de I+D, al mencionar que la capacidad de la empresa para explorar conocimientos externos es muchas veces un subproducto de sus actividades internas de I+D. A través de dichas actividades se estimularían las capacitaciones tecnológicas centrales de la empresa, lo que permitiría comprender mejor el conocimiento tácito integrado (embedded) en procesos y productos, y aumentaría sus posibilidades de acceder a los conocimientos externos y absorberlos. El concepto de que grados elevados de I+D amplían la "conectividad" de las empresas con fuentes externas de conocimiento se encuentra ampliamente difundido en diversos estudios (entre otros: Freeman, 1991; Chesbrough y Teece, 1996; Arora y Gambardella, 1994; Jensen y otros, 2007). Obviamente, tal característica no disminuye la importancia de las inversiones en I+D para la generación de conocimientos aplicados directamente a los nuevos productos y procesos, aspecto reconocido, en mayor o menor grado, en todos los estudios de economía de la innovación. 


\section{Capacidad de absorción y generación de conocimientos mediante capacitación}

La educación y la capacitación son reconocidas como importantes elementos de la capacidad de innovar de una región o un país (Lundvall y otros, 2002). A nivel de la empresa, la actividad de capacitación debe entenderse como iniciativas de transmisión de conocimientos abstractos de quien posee conocimientos especializados (know how) a aquel que, por no haber desarrollado determinada rutina específica, no los posee. No es más que una iniciativa de transmisión de conocimientos tácitos. El beneficio se plasma en el incremento de la capacidad de la empresa para descubrir y solucionar problemas surgidos en las rutinas de producción, dado que un conjunto mayor de individuos estarán aptos para ello.

Mediante la cuantificación de ese elemento se observa con frecuencia la importancia atribuida a la existencia de personal calificado dentro de la empresa. Sin embargo, conforme lo destacan Murovec y Prodan (2009), los gastos en capacitación se vinculan mucho más a las necesidades específicas definidas por las empresas. La absorción tecnológica puede ser una de esas necesidades y es precisamente tal característica la que se pretende captar mediante el indicador del "aprendizaje mediante capacitación" que se presenta a continuación.

\section{Capacidad de absorción mediante el "aprendizaje práctico"}

Arrow (1962) fue el primero en abordar la capacidad para generar nuevos conocimientos a partir del aprendizaje adquirido en las rutinas de producción. Básicamente, el autor destacó las actividades de repetición que conducen al desarrollo de habilidades productivas capaces de incrementar la productividad a través de mejoras técnicas de la manufactura. Además de la capacidad para generar nuevos conocimientos directamente aplicados a los nuevos procesos productivos, esa forma de aprender permite ampliar la capacidad de comprensión de técnicas generadas y utilizadas fuera de los límites de la empresa. Cohen y Levinthal (1990) señalan lo siguiente:

“ (...) la capacidad de absorción también puede desarrollarse como subproducto de las operaciones de manufactura de una determinada empresa. Abernathy (1978) y Rosenberg (1982) observaron que mediante la participación directa en la manufactura, una empresa está en mejores condiciones para reconocer y analizar nueva información pertinente para el mercado de un producto particular. La experiencia en la producción permite a la empresa reconocer el valor de los métodos para reorganizar o automatizar procesos de fabricación específicos e implementarlos" (Cohen y Levinthal, 1990, pág. 2). Pisano (1996) amplía ese concepto al destacar que el aspecto "práctico" puede observarse en las soluciones informáticas, los análisis de laboratorio, las pruebas de prototipos y otros experimentos no necesariamente vinculados en forma directa a la manufactura.

Sobre la base de Zahra y George (2002), surge una interpretación referida a la complementariedad que existe entre las tres formas de aprendizaje interno en el complejo proceso de absorción tecnológica. Se comprende que el aprendizaje mediante I+D estaría más vinculado a la fase i) de adquisición y ii) de asimilación de los conocimientos externos cruciales para las innovaciones de mayor repercusión en el mercado nacional, y supondría una labor a tiempo completo por parte de individuos dedicados a la comprensión y el análisis de diversos tipos de información y conocimientos externos. Las nuevas informaciones o conocimientos - comprendidos y utilizados en los laboratorios de I+D-requieren la movilización de conocimientos generados en la planta de producción, así como de aquellos ampliados gracias a la difusión de prácticas de capacitación tanto para iii) transformar el nuevo proyecto o el prototipo, respetando las especificidades de los procesos productivos de la empresa, como para iv) mejorar la capacidad de explorar los nuevos conocimientos absorbidos 9 .

\section{Nota sobre la semejanza conceptual entre capacidad de absorción y capacidad tecnológica}

En la investigación plasmada en el presente artículo se advirtió el uso de las denominaciones "capacidad de absorción" y "capacidad tecnológica" en referencia al mismo fenómeno. En esta subsección se presentan y discuten brevemente las semejanzas conceptuales observadas, aunque no de forma definitiva ni siquiera exhaustiva. El objetivo es incentivar nuevos emprendimientos académicos capaces de ampliar el rigor analítico en el uso de los términos.

En primer lugar, se observó una semejanza en los aspectos internos de la empresa que permiten incorporar

\footnotetext{
${ }^{9}$ La mayor intensidad sugerida de una de las formas de aprendizaje en cada fase del proceso de absorción no significa que no exista retroinformación en relación con las fases iniciales del proceso, incluidas nuevas consideraciones sobre el objeto de absorción tecnológica, asî como el intercambio de información entre las "fases".
} 
conocimientos externos. Desde el punto de vista de la "capacidad de absorción", los aspectos internos se manifestarían no solo en i) las rutinas organizacionales y gerenciales o en ii) la ampliación de la calificación tácita de los individuos, sino también en iii) los productos, servicios y nuevos procesos generados; mientras que la "capacidad tecnológica" estaría en i) los sistemas organizacionales; ii) el conocimiento y la calificación técnica del personal, y iii) los sistemas tecnofísicos, tales como máquinas, equipamientos, programas informáticos, plantas, manufactura y productos y servicios (Figueiredo, 2004). Las semejanzas entre i), ii) y iii) se ven complementadas por el hecho de que, en las visiones acerca de ambas capacidades, esos aspectos se configuren como capacitaciones acumuladas, dependientes de decisiones pasadas. Ello significa que, por poseer una trayectoria de aprendizaje particular, cada empresa tendría capacidades específicas para absorber conocimientos.

Una segunda semejanza consiste en la aceptación de la diversidad de fuentes internas de conocimiento que pueden ampliar tales capacitaciones. En las dos visiones, la idea del "aprendizaje práctico" (Arrow, 1962) es insuficiente para explicar la absorción de conocimientos externos. Además, se destacan la calificación de la mano de obra y la pertinencia de los niveles de I+D a nivel interno: “[...] cuando en proyectos de inversión además de la tecnología establecida se incorporan elementos de tecnología más innovadores, las capacidades necesarias podrían requerir ingeniería e I+D más sofisticados" (Bell y Pavitt, 1995, pág. 85) ${ }^{10}$.

Otra semejanza entre ambos conceptos es el reconocimiento de que los conocimientos (capacitaciones) internos y externos se combinan por medio de un proceso complejo marcado por esfuerzos en diversas etapas, entre las que incluso hay semejanza. Mientras que en el concepto de capacidad de absorción se reconoce que la etapa inicial ocurre mediante la adquisición y asimilación de los conocimientos externos (Zahra y George, 2002), en el concepto de capacidad tecnológica, la fase inicial

10 En las subsecciones 2, 3 y 4 se presentaron esos elementos en Cohen y Levinthal (1990). se vincula a la necesidad de realizar gestiones (léase inversiones) en etapas de adaptación e implementación de la nueva tecnología a las situaciones concretas en que pasará a operar.

En una fase siguiente, en lo referido a la capacidad de absorción se destaca una etapa vinculada a la capacidad de la empresa de "transformar" sus rutinas, de modo que la combinación de los nuevos conocimientos (integrados en la tecnología) con los antiguos ocurra de manera eficaz. Normalmente, sería en la fase de combinación de los conocimientos cuando surgen nuevas perspectivas y se reconocen nuevas oportunidades (Zahra y George, 2002) y, por lo tanto, tienen lugar otras modificaciones y mejoras de tecnología. En cuanto a la capacidad tecnológica, esa fase está representada en la generación de una serie de innovaciones incrementales derivadas de la adquisición de un nuevo proceso productivo. Esas innovaciones se realizarían para mantener y ampliar la competitividad de la empresa a lo largo del tiempo y se derivan y dependen de los diferenciales de capacidad tecnológica acumulados en cada empresa.

Por último, una derivación más libre de las visiones sobre capacidad de absorción y capacidad tecnológica es la comprensión de que hay semejanza en lo que se refiere al espacio territorial en que se insertan las empresas, ya que en ambos conceptos la competencia para asimilar conocimientos externos, en especial los tácitos, se deriva de características particulares del proceso de aprendizaje en que participa la empresa. Por ejemplo, al analizar los beneficios potenciales provenientes de las interacciones con proveedores, Bell y Pavitt (1995) destacan que los usuarios de materiales o componentes específicos, dotados de habilidades para transformarlos (innovar), podrían inducir activamente a sus proveedores a que se esfuercen por desarrollar esos insumos, si estos últimos estuviesen dotados de un cierto nivel de capacitación tecnológica. En ese caso, estar inserto en un Sistema Nacional de Innovación (SNI) periférico —-donde la producción puede incluir a proveedores y usuarios que no disponen de la misma capacidad técnica de aquellos vinculados al desarrollo de la tecnología localizados en los SNI maduros- puede representar una restricción importante para la difusión tecnológica, así como para la ampliación de la capacidad de absorción. 


\section{III}

\section{Patrones sectoriales de aprendizaje e indicadores de aprendizaje e innovación}

Esta sección se divide en dos subsecciones. En la primera se presentan los patrones sectoriales de aprendizaje señalados en Bittencourt (2012) y los indicadores de aprendizaje que los definieron. En la segunda se aborda la relevancia de trabajar con innovaciones de efecto mayor (para el mercado nacional) y se presenta el indicador utilizado para inferirlas.

\section{Patrones sectoriales de aprendizaje}

El uso de los patrones sectoriales de aprendizaje como referencia sectorial se justifica no solo por ser una construcción en que se consideran características del Sistema Nacional de Innovación (SNI) brasileño, sino también por el hecho de que los indicadores utilizados son adecuados para el objetivo del presente artículo.

En el cuadro 1 se relacionan los indicadores de aprendizaje elaborados en Bittencourt (2012). Las formas de aprendizaje se definieron a partir de Malerba (1992); Hedberg (1981) y Kim y Nelson (2005), teniendo en cuenta las limitaciones en términos de cuantificación impuestas por la Encuesta de Innovación Tecnológica brasileña (PINTEC, por sus siglas en portugués), realizada por el Instituto Brasileño de Geografía y Estadística (IBGE). Nótese que los indicadores permiten combinar fuentes de información utilizadas en los procesos de innovación con datos relevantes sobre los gastos en actividades de innovación, según información proporcionada por

CUADRO 1

Indicadores de aprendizaje: características conexas

\begin{tabular}{|c|c|c|c|c|c|c|c|c|c|}
\hline & & \multicolumn{8}{|c|}{ Indicadores de aprendizaje } \\
\hline & & $\begin{array}{l}1 . \\
\text { I+D a nivel } \\
\text { interno }\end{array}$ & $\begin{array}{c}2 . \\
\text { Aprendizaje } \\
\text { práctico }\end{array}$ & $\begin{array}{c}3 . \\
\text { Capacitación }\end{array}$ & $\begin{array}{c}4 . \\
\text { Ciencia y tecnología } \\
\text { avanzadas }\end{array}$ & $\begin{array}{c}5 . \\
\text { Proveedores }\end{array}$ & $\begin{array}{c}6 . \\
\text { Clientes }\end{array}$ & $\begin{array}{l}7 . \\
\text { Otras fuentes de } \\
\text { interacción }\end{array}$ & $\begin{array}{c}8 . \\
\text { Imitación }\end{array}$ \\
\hline \multirow{5}{*}{ 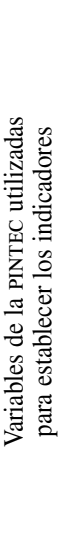 } & \multirow{4}{*}{$\stackrel{\stackrel{0}{0}}{\stackrel{0}{0}}$} & $\begin{array}{l}\text { Fuentes } \\
\text { internas de } \mathrm{I}+\mathrm{D}\end{array}$ & $\begin{array}{l}\text { Otras fuentes } \\
\text { internas }\end{array}$ & $\begin{array}{l}\text { Centros de } \\
\text { capacitación }\end{array}$ & Universidades & Proveedores & Clientes & $\begin{array}{l}\text { Conferencias, } \\
\text { encuentros y } \\
\text { publicaciones }\end{array}$ & Competidores \\
\hline & & $\cdots$ & $\cdots$ & $\cdots$ & $\begin{array}{c}\text { Institutos de } \\
\text { investigación o } \\
\text { centros tecnológicos }\end{array}$ & $\cdots$ & $\cdots$ & $\begin{array}{c}\text { Ferias y } \\
\text { exposiciones }\end{array}$ & $\begin{array}{c}\text { Licencias, } \\
\text { patentes y } \\
\text { conocimientos } \\
\text { especializados }\end{array}$ \\
\hline & & $\cdots$ & $\cdots$ & $\cdots$ & $\cdots$ & $\cdots$ & $\cdots$ & $\begin{array}{l}\text { Empresas de } \\
\text { consultoría }\end{array}$ & $\cdots$ \\
\hline & & $\cdots$ & $\cdots$ & $\cdots$ & $\cdots$ & $\cdots$ & $\cdots$ & Competidores & $\cdots$ \\
\hline &  & $\begin{array}{l}\text { I+D formal a } \\
\text { nivel interno }\end{array}$ & $\begin{array}{c}\text { Proyectos } \\
\text { industriales } \\
\text { y otras } \\
\text { preparaciones } \\
\text { técnicas }\end{array}$ & Capacitación & $\begin{array}{l}\text { Adquisición de } \\
\text { otros conocimientos } \\
\text { externos }\end{array}$ & $\begin{array}{l}\text { Adquisición } \\
\text { de máquinas y } \\
\text { equipamientos }\end{array}$ & $\begin{array}{c}\text { Introducción } \\
\text { de la } \\
\text { innovación } \\
\text { en el mercado }\end{array}$ & $\cdots$ & $\begin{array}{l}\text { Adquisición } \\
\text { de otros } \\
\text { conocimientos } \\
\text { externos }\end{array}$ \\
\hline
\end{tabular}

Características de los indicadores

Localización de la fuente del conocimiento

Dentro de la empresa

Fuera de la empresa

Característica principal del conocimiento

en cuestión

Codificado Tácito Tácito

Codificado Tácito Tácito Tácito $\begin{gathered}\begin{array}{c}\text { Codificado } \\ \text { y tácito }\end{array} \\ \text { Th }\end{gathered}$

Fuente: P.F. Bittencourt, "Padrões setoriais de aprendizagem da indústria brasileira: uma análise exploratória", Revista Brasileira de Inovação, vol. 11, $\mathrm{N}^{\circ}$ 1, Campinas, 2012.

PINTEC: Encuesta de Innovación Tecnológica brasileña. 
las empresas. En la construcción de las combinaciones se observó la probable actividad de innovación vinculada al uso de determinada fuente de información, como fuente de ideas para la innovación ${ }^{11}$. En otras palabras, se entiende que existe una gran probabilidad de que la perspectiva que surja, por ejemplo, en el departamento de I+D (fuente de información) se vincule a gastos por concepto de actividades de I+D, según la relación presentada en el Indicador 1, "aprendizaje mediante I+D a nivel interno", que se deriva del "aprendizaje mediante investigación".

Las posibles respuestas de las empresas innovadoras a las preguntas formuladas por la PINTEC y utilizadas para establecer los indicadores son siempre cualitativas: alta,

11 Sin embargo, no se parte del supuesto de que exista una correspondencia absoluta entre fuentes de ideas y los gastos en actividades de innovación. De hecho, las ideas surgidas en los departamentos de I+D (aprendizaje mediante investigación) pueden ejecutarse por medio de gastos en I+D a nivel externo (aprendizaje mediante ciencia y tecnología de alto nivel). No obstante, los indicadores estiman que existe una probabilidad mayor de que los gastos se realicen en los departamentos de I+D de la propia empresa, en ese caso. media y baja o irrelevante. Esos atributos cualitativos se transformaron en cuantitativos para que pudiese utilizarse la técnica estadística. El procedimiento consistió en reemplazar los indicadores cualitativos "alta", "media" y "baja o irrelevante" por " 1 ", " 0,66 " y " 0,167 ”, respectivamente ${ }^{12}$. Así, los indicadores se conformaron valiéndose de una media ponderada de la importancia atribuida por el conjunto de las empresas innovadoras de cada sector a las variables seleccionadas para la composición de esos indicadores. De esta manera, se aplicó a los sectores la técnica de agrupamiento del análisis estadístico multivariado (3 dígitos de la Clasificación Nacional de Actividades Económicas (CNAE)), de modo que fuesen identificados los patrones sectoriales de aprendizaje que se muestran en el cuadro 2 (Bittencourt, 2012).

12 El valor correspondiente a la especificación cualitativa "baja o irrelevante" sigue la valoración secuencial de las informaciones alta y media. El valor " 0,176 " es la media entre " 0,33 " y " 0,00 ", valores atribuidos a "baja" e "irrelevante", respectivamente.

\section{Sectores que componen los patrones sectoriales de aprendizaje}

\begin{tabular}{|c|c|c|c|}
\hline $\begin{array}{c}\text { Patrón 1 } \\
\text { Sectores intensivos en } \\
\text { aprendizaje en la esfera } \\
\text { productiva }\end{array}$ & $\begin{array}{l}\text { Patrón } 2 \\
\text { Sectores intensivos en } \\
\text { aprendizaje en etapas } \\
\text { tempranas }\end{array}$ & $\begin{array}{c}\text { Patrón } 3 \\
\text { Sectores intensivos en } \\
\text { múltiples formas } \\
\text { de aprendizaje }\end{array}$ & $\begin{array}{c}\text { Patrón } 4 \\
\text { Sectores intensivos en } \\
\text { aprendizaje interno y } \\
\text { en etapas posteriores }\end{array}$ \\
\hline Carne y pescado & $\begin{array}{l}\text { Extracción de piedra, arena y } \\
\text { arcilla }\end{array}$ & Productos farmacéuticos & Químicos inorgánicos \\
\hline $\begin{array}{l}\text { Aceites de origen } \\
\text { vegetal y animal }\end{array}$ & Extracción de otros minerales & Plaguicidas agrícolas & $\begin{array}{l}\text { Resinas, elastómeros, fibras, } \\
\text { filamentos artificiales y } \\
\text { sintéticos }\end{array}$ \\
\hline Lácteos & $\begin{array}{l}\text { Molienda, fabricación de } \\
\text { amiláceos (contienen almidón) } \\
\text { y de raciones }\end{array}$ & $\begin{array}{l}\text { Máquinas y equipamientos } \\
\text { para informática y oficina }\end{array}$ & $\begin{array}{l}\text { Tintas, barnices, esmaltes, } \\
\text { lacas y productos afines }\end{array}$ \\
\hline Azúcar & Productos alimenticios & $\begin{array}{l}\text { Hilos, cables y conductores } \\
\text { eléctricos aislados }\end{array}$ & $\begin{array}{l}\text { Productos y preparados } \\
\text { químicos diversos }\end{array}$ \\
\hline Café & Fibras textiles naturales & $\begin{array}{l}\text { Pilas, baterías y acumuladores } \\
\text { eléctricos }\end{array}$ & $\begin{array}{l}\text { Artículos de cuchillería, } \\
\text { herrería y herramientas } \\
\text { manuales }\end{array}$ \\
\hline Bebidas & Acabado en hilos y tejidos & $\begin{array}{l}\text { Aparatos y equipamientos de } \\
\text { telefonía y radiotelefonía, y } \\
\text { de transmisores de televisión } \\
\text { y radio }\end{array}$ & $\begin{array}{l}\text { Motores, bombas, compresores } \\
\text { y equipamientos de } \\
\text { transmisión }\end{array}$ \\
\hline Hilado & $\begin{array}{l}\text { Artefactos de tejidos y } \\
\text { de otros textiles }\end{array}$ & $\begin{array}{l}\text { Aparatos e instrumentos de } \\
\text { medida, prueba y control }\end{array}$ & $\begin{array}{l}\text { Máquinas y equipamientos de } \\
\text { uso general }\end{array}$ \\
\hline Celulosa & Tejidos de punto & $\begin{array}{l}\text { Aparatos, instrumentos y } \\
\text { materiales ópticos, fotográficos } \\
\text { y cinematográficos }\end{array}$ & $\begin{array}{l}\text { Máquinas y equipamiento de } \\
\text { usos en la extracción mineral y } \\
\text { construcción }\end{array}$ \\
\hline Alcohol & $\begin{array}{l}\text { Confección de vestuario y } \\
\text { accesorios }\end{array}$ & $\begin{array}{l}\text { Automóviles, camionetas y } \\
\text { utilitarios }\end{array}$ & $\begin{array}{l}\text { Otras máquinas y } \\
\text { equipamiento de uso en la } \\
\text { extracción mineral }\end{array}$ \\
\hline
\end{tabular}


Cuadro 2 (conclusión)

\begin{tabular}{|c|c|c|c|}
\hline $\begin{array}{c}\text { Patrón 1 } \\
\text { Sectores intensivos en } \\
\text { aprendizaje en la esfera } \\
\text { productiva }\end{array}$ & $\begin{array}{c}\text { Patrón } 2 \\
\text { Sectores intensivos en } \\
\text { aprendizaje en etapas } \\
\text { tempranas }\end{array}$ & $\begin{array}{c}\text { Patrón } 3 \\
\text { Sectores intensivos en } \\
\text { múltiples formas } \\
\text { de aprendizaje }\end{array}$ & $\begin{array}{c}\text { Patrón } 4 \\
\text { Sectores intensivos en } \\
\text { aprendizaje interno y } \\
\text { en etapas posteriores }\end{array}$ \\
\hline Cemento & $\begin{array}{l}\text { Vestuario de seguridad } \\
\text { profesional }\end{array}$ & Camiones y ómnibus & $\begin{array}{l}\text { Material eléctrico para } \\
\text { vehículos }\end{array}$ \\
\hline Hierro colado y ferroaleaciones & $\begin{array}{l}\text { Artículos para viaje y de } \\
\text { artefactos de cuero }\end{array}$ & Productos del tabaco & $\begin{array}{l}\text { Fabricación y reparación } \\
\text { de máquinas, aparatos y } \\
\text { materiales eléctricos }\end{array}$ \\
\hline Tubos & Calzados & & Material electrónico básico \\
\hline Metalurgia de metales no férreos & Troceado y despiece de madera & & $\begin{array}{l}\text { Otros equipamientos de } \\
\text { transporte }\end{array}$ \\
\hline Productos diversos de metal & $\begin{array}{l}\text { Productos de madera y } \\
\text { material trenzado }\end{array}$ & & $\begin{array}{l}\text { Productos derivados del } \\
\text { petróleo }\end{array}$ \\
\hline $\begin{array}{l}\text { Tractores, máquinas y } \\
\text { equipamientos para agricultura }\end{array}$ & $\begin{array}{l}\text { Embalajes de papel o cartón } \\
\text { ondulado }\end{array}$ & & $\begin{array}{l}\text { Armas, municiones y } \\
\text { equipamientos militares }\end{array}$ \\
\hline Máquinas-herramientas & $\begin{array}{l}\text { Papel, cartón ondulado, } \\
\text { cartulina y cartón }\end{array}$ & & $\begin{array}{l}\text { Construcción y reparación de } \\
\text { embarcaciones y vehículos } \\
\text { ferroviarios }\end{array}$ \\
\hline Equipos de energía eléctrica & $\begin{array}{l}\text { Edición, impresión y } \\
\text { reproducción }\end{array}$ & & $\begin{array}{l}\text { Construcción, montaje y } \\
\text { reparación de aeronaves }\end{array}$ \\
\hline Autopartes & $\begin{array}{l}\text { Productos químicos orgánicos } \\
\text { Artículos de caucho } \\
\text { Productos de material plástico } \\
\text { Vidrio y productos del vidrio } \\
\text { Artefactos de concreto, } \\
\text { cemento y materiales similares } \\
\text { Productos cerámicos } \\
\text { Pulido de piedras y fabricación } \\
\text { de cal, entre otros } \\
\text { Fundición } \\
\text { Estructuras metálicas y obras } \\
\text { de calderería pesada } \\
\text { Tanques, calderas, depósitos } \\
\text { metálicos } \\
\text { Servicios de pulvimetalurgia y } \\
\text { tratamiento de metales } \\
\text { Mantenimiento y reparación de } \\
\text { máquinas y equipamientos } \\
\text { Fabricación de lámparas y } \\
\text { equipamientos de iluminación } \\
\text { Cabinas, carrocerías y } \\
\text { recuperación de motores } \\
\text { Artículos del mobiliario } \\
\text { Productos diversos } \\
\text { Reciclaje }\end{array}$ & & \\
\hline
\end{tabular}

Fuente: P.F. Bittencourt, "Padrões setoriais de aprendizagem da indústria brasileira: uma análise exploratória", Revista Brasileira de Inovação, vol. 11, N 1, Campinas, 2012.

\section{Innovaciones para el mercado nacional}

En la PINTEC se definen dos grados de innovación: para la empresa y para el mercado nacional. Como se muestra en el cuadro 3, las innovaciones de grado superior son poco frecuentes y su porcentaje disminuyó desde la primera encuesta. La baja frecuencia se debe a factores que caracterizan al precario desarrollo histórico del SNI brasileño (Albuquerque, 2000; Viotti, 2002).

En el presente trabajo el enfoque se centra en la parte restringida de innovaciones para el mercado nacional, por i) poseer mayor repercusión en la dinámica de desarrollo económico, y ii) en comparación con las innovaciones para la empresa, requerir un conjunto más amplio de conocimientos para su realización.

Para medir las innovaciones de gran repercusión entre los sectores de la actividad económica se utilizó como indicador el porcentaje de esas innovaciones en relación con el total de ellas registradas en el sector en cada período. Las correlaciones calculadas entre este indicador y los de aprendizaje solo fueron posibles por tratarse de medidas de intensidad. 
Brasil: innovaciones para la empresa y para el mercado nacional

\begin{tabular}{|c|c|c|c|c|c|c|}
\hline \multirow{2}{*}{ Año } & \multirow{2}{*}{$\begin{array}{l}\text { Número de } \\
\text { empresas } \\
\text { innovadoras }\end{array}$} & \multirow{2}{*}{$\begin{array}{c}\text { Tasa de } \\
\text { innovación }\end{array}$} & \multicolumn{2}{|c|}{$\begin{array}{l}\text { Porcentaje de innovaciones } \\
\text { de producto }\end{array}$} & \multicolumn{2}{|c|}{$\begin{array}{l}\text { Porcentaje de innovaciones } \\
\text { de proceso }\end{array}$} \\
\hline & & & Empresa & Mercado nacional & Empresa & Mercado nacional \\
\hline 2000 & 22698 & 31,52 & 32,28 & 9,27 & 52,22 & 6,23 \\
\hline 2003 & 28036 & 33,27 & 37,62 & 5,67 & 54,18 & 2,53 \\
\hline 2005 & 30377 & 33,56 & 35,42 & 6,90 & 54,15 & 3,52 \\
\hline
\end{tabular}

Fuente: primera, segunda y tercera Encuesta de Innovación Tecnológica (PINTEC).

\section{IV}

\section{Metodología}

La metodología se divide de la siguiente forma: en la subsección 1 da a conocer el tratamiento estadístico aplicado a los datos, o sea, las correlaciones simples y parciales y los modelos de causalidad estadística que orientan el análisis. En la número 2 se interpretan esos modelos. En la 3 se destaca el objeto de la investigación. Por último, en la subsección 4 se presentan las condiciones estadísticas necesarias para el estudio de los casos.

\section{Modelos de causalidad estadística mediante la correlación de Pearson}

Los coeficientes de correlación de Pearson " $r$ " se aplican ampliamente en las ciencias económicas como medidas de dependencia lineal entre dos variables. Varían entre -1 (correlación negativa perfecta) y 1 (correlación positiva perfecta). Formalmente el coeficiente es dado por:

$$
\begin{gathered}
\rho=\frac{\sum_{i=1}^{n}\left(x_{i}-\bar{x}\right)\left(y_{i}-\bar{y}\right)}{\sqrt{\sum_{i=1}^{n}\left(x_{i}-\bar{x}\right)^{2}} \cdot \sqrt{\sum_{i=1}^{n}\left(y_{i}-\bar{y}\right)^{2}}} \\
\frac{\operatorname{cov}(X, Y)}{\sqrt{\operatorname{var}(X) \cdot \operatorname{var}(Y)}}
\end{gathered}
$$

siendo que:

$$
\bar{x}=\frac{1}{n} \cdot \sum_{i=1}^{n} x_{i} \text { y } \bar{y}=\frac{1}{n} \cdot \sum_{i=1}^{n} y_{i}
$$

son las medias aritméticas de las variables.

Los coeficientes de correlación parcial sirven para medir cuál sería la intensidad de la correlación entre dos indicadores determinados — ' $\mathrm{B}$ ' y ' $\mathrm{C}$ ' - si otro indicador, digamos ' $\mathrm{A}$ ', hipotéticamente, influenciase tanto a 'B' como a 'C', y permiten medir la intensidad de la correlación entre ' $\mathrm{B}$ ' y ' $\mathrm{C}$ ' después de descontar los efectos de 'A'.

En el presente artículo, 'A' se refiere a los indicadores de aprendizaje interno, ' $\mathrm{B}$ ' al de aprendizaje a través de clientes y ' $C$ ' al indicador de intensidad de las innovaciones de producto para el mercado nacional (véase el cuadro 4).

Esa metodología es adecuada porque define diferentes relaciones de causa entre A, B y C a través de los coeficientes de correlación simples y parciales (Legendre y Legendre, 1998). Tanto en el artículo de estos autores como en las estadísticas, la causalidad concierne a la hipótesis de que los cambios en un indicador (variable) tienen un efecto en los cambios en otro indicador (variable).

Una desventaja potencial que supone la estimación de intervalos de confianza para la correlación de Pearson " $r$ " es la suposición de distribución normal bivariada entre X e Y. Esa desventaja se evitó al estimar los intervalos de confianza mediante la técnica de bootstrap con 5.000 nuevos muestreos aleatorios. El uso de dicha técnica no provoca transformaciones en los resultados de los cálculos de los coeficientes de Pearson, sino en los intervalos de confianza, lo que permite no asumir la binormalidad típica de la prueba de hipótesis tradicional (Efron y Tibshirani, 1993). Los resultados de la técnica están disponibles en Bittencourt (2010) ${ }^{13}$.

13 Respecto de cada matriz de correlación de Pearson se presenta una banda inferior y una banda superior de las posibilidades. Esas bandas son los parámetros que permiten rechazar o no la hipótesis nula, es decir, de que la correlación es diferente de cero. En el presente estudio, solo las correlaciones positivas revisten importancia. Para ellas, una banda inferior negativa no permite rechazar la hipótesis de que la correlación de Pearson es estadísticamente diferente de cero. 
CUADRO 4

Indicadores de aprendizaje e innovación correspondientes

a los modelos causales

\begin{tabular}{|c|c|c|c|}
\hline Variables de los modelos causales & A & B & $\mathrm{C}$ \\
\hline & $\begin{array}{l}\text { Aprendizaje interno. } \\
\text { Fuentes internas de generación } \\
\text { de conocimientos }\end{array}$ & $\begin{array}{l}\text { Aprendizaje externo. } \\
\text { Fuentes externas de absorción } \\
\text { tecnológica }\end{array}$ & $\begin{array}{l}\text { Desempeño. Porcentaje de } \\
\text { innovaciones para el mercado } \\
\text { nacional }\end{array}$ \\
\hline $\begin{array}{l}\text { Indicadores de aprendizaje interno, } \\
\text { externo y desempeño innovador }\end{array}$ & $\begin{array}{l}\text { - I+D a nivel interno } \\
\text { - Capacitación } \\
\text { - Aprendizaje práctico }\end{array}$ & $\begin{array}{l}\text { - Universidades } \\
\text { - Proveedores } \\
\text { - Clientes } \\
\text { - Conferencias, encuentros } \\
\text { y publicaciones } \\
\text { - Competidores }\end{array}$ & Producto \\
\hline
\end{tabular}

Fuente: elaboración propia.

I+D: investigación y desarrollo.

En el gráfico 1 se presentan los modelos causales y las condiciones que cumplirán los índices de correlación simple y parcial entre las variables 'A', 'B' y ' $C$ '. El modelo 1 se denomina "modelo de efecto indirecto"; el modelo 2, "modelo de doble causa" y el modelo 3, "modelo de múltiple causalidad". Estos se interpretan en la secuencia.

Modelo 1. Señala la presencia de efecto indirecto de causa de A a C, intermediado por B. Según los casos que explica ese modelo, los conocimientos generados en A (aprendizaje interno) posibilitan la absorción de aquellos presentes en B (aprendizaje externo), que generan C (innovaciones). Las innovaciones se sustentan ampliamente en la capacidad de absorción.

Modelo 2. Indica que tanto el efecto de absorción tecnológica de A hacia B, como el efecto de generación de nuevos conocimientos de $\mathrm{A}$ hacia $\mathrm{C}$, están presentes (doble efecto), mientras que la absorción tecnológica solo es efectiva en presencia de (A). Ello se debe a que, descontada la influencia de A, la correlación parcial rbc.a no es significativamente diferente de cero. La correlación simple $r$.bc depende de la existencia de A. En otras palabras, la absorción del conocimiento de los externos depende en gran medida de la forma de aprendizaje interno objeto de análisis.

Modelo 3. También entre los casos revelados por ese modelo está presente el doble efecto de las formas internas de aprendizaje. Sin embargo, a diferencia del modelo 2 , la correlación $r$ bc.a difiere significativamente de cero, lo que indica que una parte importante de los conocimientos externos absorbidos es independiente de la forma de aprendizaje interna (A) objeto de análisis. O sea, incluso en la ausencia de (A), los conocimientos

GRÁFICO 1

Modelos de causalidad estadística

\begin{tabular}{|c|c|c|c|}
\hline Modelo & 1 & 2 & 3 \\
\hline Causalidad & $\begin{array}{l}\mathrm{A} \\
\downarrow \\
\mathrm{B} \\
\downarrow\end{array}$ & $\nu_{\mathrm{B}}^{\mathrm{A}}$ & ${ }_{\mathrm{B}}^{\mathrm{A}} \mathrm{C}_{\mathrm{C}}$ \\
\hline Condiciones & $\begin{array}{c}r \mathrm{ab}, r \mathrm{bc}, \text { rab.c y } r \mathrm{bc} . \mathrm{a} \neq 0 \\
r \text { ac.b no significativa } \\
|r a b|>|r a c| \\
|r \mathrm{bc}|>|r a c| \\
|r a b . c|<|r a b| \\
|r b c . a|<|r b c|\end{array}$ & $\begin{array}{c}r a b, r a c, \text { rab.c, } r a c . b \neq 0 \\
\text { rbc.a no significativa } \\
|r a b|>|r b c| \\
|r a c|>|r b c| \\
|r a b . c|<|r a b| \\
|r a c . b|<|r a c|\end{array}$ & $\begin{array}{c}r \mathrm{ab}, r \mathrm{bc}, r \mathrm{ac} \neq 0 \\
r \mathrm{ab} . \mathrm{c}, r \mathrm{bc} \cdot \mathrm{a}, r \mathrm{ac} . \mathrm{b} \neq 0\end{array}$ \\
\hline
\end{tabular}

Fuente: elaboración propia sobre la base de P. Legendre y L. Legendre, Numerical Ecology, Amsterdam, Elsevier, 1998. 
generados en (B) se absorben para generar (C). En comparación con el modelo 2 , en este caso la absorción del conocimiento externo depende en menor medida de la forma de aprendizaje interno.

\section{Enfoque de la investigación: absorción tecnológica en los patrones sectoriales}

Según se mencionó anteriormente, el indicador que mide la intensidad de innovaciones para el mercado nacional es su porcentaje en relación con el total de innovaciones registradas en el sector en cada período.

Considerando las relaciones posibles entre (A) conocimientos internos de la empresa, (B) conocimientos externos a la empresa, y (C) innovaciones de producto para el mercado nacional, en los cuatro patrones sectoriales de aprendizaje se observó que podrían investigarse más de 50 posibilidades ${ }^{14}$. El siguiente procedimiento permitió reducir el número de las aplicaciones.

A partir del concepto teórico de que el acceso a la información externa es el primer indicador de que las empresas están absorbiendo conocimientos (Schmidt, 2005; De Negri, 2006), se excluyeron de la investigación las correlaciones entre la intensidad de las innovaciones de producto para el mercado nacional (c) y las formas externas de aprendizaje (b) que no fuesen

${ }^{14}$ Estrictamente, serían 60 posibilidades, como resultado de multiplicar $4 \times 3 \times 5$, que corresponde, respectivamente, al número de patrones de aprendizaje (4), a las formas internas de aprendizaje (3) y a las formas externas de aprendizaje (5). positivas y significativamente diferentes de cero. Solo las correlaciones que suponen el uso de los clientes cumplieron esa condición y únicamente entre los sectores de los patrones 1, 2 y 3 . Las correlaciones simples fueron, respectivamente, $r=0,40, r=0,44$ y $r=0,33^{15}$.

De ese procedimiento se derivaron nueve posibilidades de investigación de la ampliación de la capacidad de absorción derivada de los esfuerzos internos, como se resume en el cuadro 5.

Por lo general, los clientes absorben conocimientos mediante prácticas de adaptación de los productos a la demanda, lo que supone una interacción entre productor y usuario capaz de ampliar la comprensión de uno sobre las necesidades del otro. Los contactos personales ocurren sobre todo al final de los procesos de innovación, incluso en la planta de producción, cuando se necesitan etapas de adaptaciones técnicas. Estos contactos pueden considerar pruebas de mercado y adaptaciones del producto a diferentes mercados.

En lo referido a la absorción de los conocimientos de los clientes para producir innovaciones de producto

\footnotetext{
15 En los sectores del patrón 4, con el método utilizado no se identifica la absorción tecnológica debido a la inexistencia de correlación simple positiva y significativamente diferente de cero entre cualquier forma de aprendizaje externo y las innovaciones analizadas. Cabe agregar que los proveedores y las fuentes avanzadas de ciencia y tecnología (CyT) presentaron correlaciones significativas a las innovaciones de proceso para el mercado nacional. También el uso de fuentes de "imitación", fuentes externas de proveedores y clientes, se correlacionó significativamente con las innovaciones de la empresa, tanto con respecto a los productos como a los procesos.
}

\begin{tabular}{llll}
\hline Patrón & Enfoques de investigación & & $\begin{array}{c}\text { Absorción tecnológica respecto de la innovación } \\
\text { de producto para el mercado nacional }\end{array}$ \\
\cline { 3 - 4 } & de la capacidad de absorción & Aprendizaje interno & Aprendizaje externo \\
\hline Patrón 1 & Investigación 1 & I+D a nivel interno & Clientes \\
Sectores intensivos en aprendizaje en & Investigación 2 & Aprendizaje práctico & Clientes \\
Patrón 2 & Investigación 3 & Capacitación & Clientes \\
Sectores intensivos en aprendizaje en & Investigación 4 & I+D a nivel interno & Clientes \\
etapas tempranas & Investigación 5 & Aprendizaje práctico & Clientes \\
Patrón 3 & Investigación 6 & Capacitación & Clientes \\
Sectores intensivos en múltiples & Investigación 7 & I+D a nivel interno & Clientes \\
formas de aprendizaje & Investigación 8 & Aprendizaje práctico & Clientes \\
& Investigación 9 & Capacitación & Clientes
\end{tabular}

Fuente: elaboración propia.

I+D: investigación y desarrollo. 
derivadas de i) actividades de I+D a nivel interno; ii) capacitación de personal, y iii) aprendizaje práctico, se destaca que i) el carácter experimental de algunas actividades de $\mathrm{I}+\mathrm{D}$, debido a que dependen de conocimientos de los clientes, puede explicar la relación; ii) los conocimientos del aprendizaje práctico pueden considerar la interacción con clientes cuando los ensayos, las pruebas, la formulación de especificaciones técnicas y la mejora de características operacionales de los productos (no incluidos en las rutinas de I+D) resultan necesarios o pertinentes, y iii) las prácticas de capacitación, por referirse a la difusión de conocimientos dentro de la empresa, que se suman a los conocimientos que los individuos ya poseían, amplían las posibilidades de descubrir potencialidades y mejorar nuevos productos.

\section{Condiciones estadísticas necesarias para la investigación de los casos seleccionados}

Además de la condición básica de que exista una correlación positiva y significativamente diferente de cero entre (b) y (c), se impusieron otras con el objetivo de limitar la investigación a los casos efectivamente pertinentes para el propósito del trabajo. Ellas son:

i) La correlación entre A y B (ra.b) debe ser positiva y es necesario que sea significativamente diferente de cero. Esta correlación indica que los conocimientos absorbidos de una fuente externa (B) se vinculan a una determinada forma interna de aprendizaje (A). Las correlaciones negativas indicarían que el uso de fuentes internas (I+D, capacitación y aprendizaje práctico) se correlaciona inversamente con una de las formas de aprendizaje externo (clientes y proveedores, entre otros). Esas relaciones no están consideradas en el espectro del presente trabajo.

ii) La correlación entre A y C ( $r a c)$ debe ser positiva, pero no es necesario que sea significativamente diferente de cero, ya que las relaciones causales indirectas entre $\mathrm{A}$ y $\mathrm{C}$ pueden ser reveladas. Sin embargo, las correlaciones negativas indicarían relaciones inversas entre $\mathrm{A}$ y $\mathrm{C}$, que no se pretende explicar.

iii) La correlación parcial entre $\mathrm{B}$ y $\mathrm{C}$, sin contar la influencia de A (rbc.a), debe ser menor que la correlación entre B y C. Eso significa que (A) es un elemento determinante de la correlación $r$ bc. Cuando A está presente, la correlación entre rbc es mayor, de lo que se deriva que los conocimientos generados en (A) sirven al objetivo de absorción de B, pero también a la generación de C. Ese indicador, vinculado a las condiciones 1 y 2 señaladas anteriormente, es suficiente para confirmar la hipótesis de que el aprendizaje interno generado en (A) influye en la absorción de conocimientos externos (B) para la generación de un tipo de innovación (C).

\section{V}

\section{Resultados y discusión}

En esta sección se discuten los resultados de la aplicación de la metodología. El enfoque de la discusión en los patrones sectoriales de aprendizaje permite señalar cuáles son las formas de aprendizaje interno que mejor explican la absorción tecnológica en cada patrón sectorial de aprendizaje en el Brasil.

\section{Innovaciones de producto para el mercado nacional y capacidad de absorción}

Los resultados de la aplicación de la metodología se resumen en el cuadro 6 que figura a continuación. Además del enfoque de la investigación por patrón sectorial de aprendizaje, que ya se destacó en el cuadro 5 , en el cuadro 6 se presentan los valores de las correlaciones estadísticas necesarias para la investigación (condiciones) y el resultado de esta. En la última columna se revela que tan solo se confirmaron seis de los nueve enfoques de investigaciones.

El enfoque 2 de la investigación (I-2) no se confirmó, pues la correlación entre el aprendizaje práctico (a) y el uso de los clientes (b) $r a b=0,06$ no fue significativamente diferente de cero. El enfoque (I-3) no se confirmó, pues la correlación simple entre capacitación (a) y las innovaciones de producto (c) $r \mathrm{ac}=-0,14$ fue negativa. El enfoque (I-9) no se confirmó, pues además de que la correlación $r$ ac $=0,04$ es muy baja, la influencia del aprendizaje mediante capacitación (a) sobre la correlación $r \mathrm{bc}$ fue negativa.

En el modelo 3 se confirmó el enfoque de la investigación 1 (I-1). En este modelo se sugiere la presencia del doble efecto de las actividades de I+D a 


\begin{tabular}{|c|c|c|c|c|c|c|c|c|}
\hline \multirow{2}{*}{ Patrón } & \multirow{2}{*}{$\begin{array}{l}\text { Enfoques de la } \\
\text { investigación }\end{array}$} & \multicolumn{2}{|c|}{$\begin{array}{l}\text { Innovación de producto para el } \\
\text { mercado nacional }(\mathrm{C})\end{array}$} & \multicolumn{4}{|c|}{ Condiciones } & \multirow{2}{*}{$\begin{array}{c}\text { Resultado } \\
\text { de la investigación }\end{array}$} \\
\hline & & $\begin{array}{l}\text { Aprendizaje interno } \\
\text { (A) }\end{array}$ & $\begin{array}{l}\text { Aprendizaje } \\
\text { externo (B) }\end{array}$ & $r a b$ & rac & $r b c$ & rbc.a & \\
\hline \multirow[t]{3}{*}{$P-1$} & I - 1 & I+D a nivel interno & Clientes & 0,39 & 0,23 & 0,40 & 0,35 & Confirmada: $\mathrm{M}^{\mathrm{a}}-3$ \\
\hline & $I-2$ & Aprendizaje práctico & Clientes & 0,06 & 0,26 & 0,40 & 0,39 & No confirmada: rab no significativa \\
\hline & $\mathrm{I}-3$ & Capacitación & Clientes & $(0,06)$ & $(0,14)$ & 0,40 & 0,39 & No confirmada: rac negativa \\
\hline \multirow[t]{3}{*}{$P-2$} & $\mathrm{I}-4$ & I+D a nivel interno & Clientes & 0,46 & 0,48 & 0,44 & 0,29 & Confirmada: M - 3 \\
\hline & I - 5 & Aprendizaje práctico & Clientes & 0,19 & 0,21 & 0,44 & 0,42 & Confirmada: M - 3 \\
\hline & I - 6 & Capacitación & Clientes & 0,22 & 0,12 & 0,44 & 0,33 & Confirmada: M - 1 \\
\hline \multirow[t]{2}{*}{$P-3$} & $\mathrm{I}-7$ & I+D a nivel interno & Clientes & 0,58 & 0,52 & 0,33 & 0,03 & Confirmada: M - 2 \\
\hline & I - 8 & Aprendizaje práctico & Clientes & 0,58 & 0,21 & 0,33 & 0,25 & Confirmada: $\mathrm{M}$ - 1 \\
\hline
\end{tabular}

Fuente: elaboración propia.

a $\mathrm{M}$ - Modelo.

I+D: investigación y desarrollo.

nivel interno (Cohen y Levinthal, 1989). La observación del valor de las correlaciones $r a b=0,39$ combinada con la $r$ bc. $a=0,35$ sugiere, sin embargo, una influencia relativamente limitada de las actividades de I+D en la absorción de conocimientos de los clientes (B) generadores de innovaciones $(C)(r b c=0,40)$. En otras palabras, se entiende que, incluso ante la ausencia de I+D, los conocimientos pertinentes de los clientes se transferirían a las empresas ${ }^{16}$.

En el análisis de los sectores del patrón sectorial 2, se confirmaron los tres enfoques de la investigación:

I-4 a través del modelo 3, en el que se destaca el doble efecto de las actividades de I+D a nivel interno. Nuevamente, el hecho de que la correlación parcial $r$ bc.a $=0,29$ sea considerablemente menor que $r \mathrm{bc}=0,44$, sugiere que otras formas de aprendizaje interno pueden influir en la capacidad de absorción.

Ello se reveló en la confirmación tanto de I-5 como de I-6, mediante el modelo 3 y el modelo 1 , respectivamente. En el análisis de I-5 se sugiere un efecto menor del aprendizaje práctico en relación con las actividades de I+D a nivel interno, tanto de su potencial de ampliación de la capacidad de absorción, como de la aplicación directa de conocimientos generadores de innovaciones. Esa interpretación se basa, respectivamente, en la correlación $r$ bc.a $=0,42$ que indica una influencia

16 La combinación de los resultados de (I-1, I-2 e I-3) configura la primera evidencia de la necesidad de ampliar los estudios sobre la absorción tecnológica. Concretamente, en el sentido de investigar más variables del comportamiento interno de la empresa capaces de cuantificar la capacidad de absorción de conocimientos externos. muy limitada de (a) sobre $r b c=0,44$, así como en la correlación $r a b=0,19$, que fue considerablemente menor que aquella verificada en el caso de las actividades de $\mathrm{I}+\mathrm{D}$ a nivel interno $r \mathrm{ab}=0,46$.

I-6 se confirmó mediante el modelo 1, lo que sugiere efectos exclusivamente indirectos de las actividades de capacitación en la generación de innovaciones. En el análisis más minucioso se sugiere que las actividades de capacitación tienen un bajo nivel de influencia, aunque no despreciable, en la ampliación de la absorción de conocimientos de los clientes. Esa interpretación surge a partir del siguiente conjunto de resultados: $r a c=0,12$, no es significativamente diferente de cero, lo que excluye una vinculación directa entre las actividades de capacitación y la generación de innovaciones; $r a b=0,22$, es significativamente diferente de cero, lo que sugiere que la absorción de conocimientos de los clientes derivada del aprendizaje mediante capacitación no es despreciable, y $r \mathrm{bc}=0,44$ en combinación con $r b c . a=0,33$ sugieren poca influencia del aprendizaje mediante capacitación en la absorción de conocimiento de los clientes.

I-7, que se confirmó mediante el modelo 2, revela que, entre los sectores de alta dinámica de aprendizaje (patrón 3), la absorción de los conocimientos de los clientes depende en gran medida de la realización de actividades de $\mathrm{I}+\mathrm{D}$ a nivel interno. Posiblemente, ello proviene del nivel de exigencia y de la complejidad de los conocimientos que suponen las ventas de esos sectores, estrechamente vinculados a las actividades tecnológicas del actual paradigma. Tal interpretación se deriva de la presencia de las actividades de I+D a nivel 
interno como un poderoso factor de confusión de la correlación entre la absorción de los conocimientos de los clientes y la generación de innovaciones de producto $(r b c=0,33)$, lo que se muestra en la correlación parcial $r \mathrm{bc} . \mathrm{a}=0,03$.

Incluso en el patrón 3, I-8 -que se vincula al aprendizaje práctico- se confirmó a través del modelo 1, lo que sugiere una relación exclusivamente indirecta entre el aprendizaje práctico e innovaciones de producto de gran efecto. En ese caso, la influencia de (a) en la correlación $r \mathrm{bc}$ se redujo, y pasó de $r \mathrm{bc}=0,33 \mathrm{a}$ $r$ bc. $\mathrm{a}=0,25$. Es importante notar que a partir de los resultados de I-7 se podría presumir que, además de las actividades de I+D, no habría más espacio para la influencia de cualquier otra forma de aprendizaje interno en la correlación $r b c$, ya que se habría tornado prácticamente nula. No obstante, se debe recordar que la intensidad en cuanto a I+D a nivel interno no excluye la intensidad en el "aprendizaje práctico", dado que las entrevistas de la PINTEC permiten que se registre más de una fuente interna de aprendizaje como pertinente para el proceso de innovación. Así, sectores intensivos en I+D a nivel interno pueden también ser intensivos en el "aprendizaje práctico". De esa forma, en el análisis conjunto de I-7 e I-8 se sugiere que existe una complementariedad entre los conocimientos generados por las dos formas de aprendizaje y la absorción de los conocimientos de los clientes capaces de generar innovaciones que repercuten en el mercado nacional.

Según los resultados obtenidos, se advierte que en el proceso de adquisición, asimilación y transformación

\section{VI}

\section{Conclusiones}

Antes de presentar las reflexiones finales, se destacan algunas limitaciones del trabajo. En primer lugar, se debe enfatizar que el uso de los sectores como referencia de los análisis supuso admitir homogeneidad en cuanto al comportamiento de las empresas, lo que ciertamente es algo poco riguroso teóricamente y tan solo permite ofrecer "evidencias" estadísticas con respecto a la absorción tecnológica o cualquier otro fenómeno. Además, ese factor implicó trabajar con un número reducido de observaciones, lo que representa un limitante estadístico, ya que restringe los grados de libertad concernientes a la exploración cuantitativa. Ello de las informaciones externas, constituye un elemento decisivo saber "quiénes" son los detentadores de los conocimientos útiles que pueden transformarse en innovaciones de gran repercusión, por lo menos entre los sectores incluidos en los patrones 1, 2 y 3 . Sin embargo, el conjunto de conocimientos desarrollados internamente que hacen viable tal absorción fue diferente entre los patrones sectoriales de aprendizaje.

Entre los sectores definidos por la intensidad del uso de fuentes de la esfera productiva (patrón 1), las actividades internas de I+D fueron el principal inductor de la capacidad de absorción de conocimientos de los clientes, útiles para generar innovaciones de efecto mayor.

Entre los sectores del patrón 2, definidos por el aprendizaje en etapas tempranas, no solo las actividades de I+D a nivel interno, sino también los conocimientos derivados de las prácticas de capacitación y del "aprendizaje práctico" se vinculan a la absorción de conocimientos de los clientes. Sectores más intensivos en las tres formas de aprender se diferencian, por lo tanto, por la capacidad de tener acceso a conocimientos de sus clientes y generar innovaciones de producto de alta repercusión.

Entre los sectores definidos por las múltiples formas de aprendizaje (patrón 3), la absorción de conocimientos de los clientes, que actúa como determinante de las innovaciones para el mercado nacional, supone conocimientos complementarios adquiridos a través de las actividades de I+D a nivel interno y del aprendizaje derivado de las rutinas internas de manufactura ("aprendizaje práctico"). puede explicar, por ejemplo, el hecho de que no se ha verificado la esperada ${ }^{17}$ significancia de correlaciones simples entre el uso de fuentes avanzadas de ciencia y tecnología y las innovaciones de producto para el mercado nacional.

\footnotetext{
17 Expectativas como esas se basan en la composición sectorial de los patrones de aprendizaje, concretamente, en su vinculación con la taxonomía de Pavitt (1984). Se esperaba, por ejemplo, encontrar evidencias de la ampliación de la absorción de conocimientos de los clientes por medio de los esfuerzos del "aprendizaje práctico" entre los sectores del patrón 4, cuya composición sectorial guarda similitudes con los "proveedores especializados" (Pavitt, 1984).
} 
Asimismo, entre los principales resultados se observó que los indicadores utilizados son insuficientes para sacar conclusiones sobre la capacidad de absorción. Una línea de investigación recomendable, en ese sentido, sería la de explorar características de la fuerza de trabajo de las empresas.

Empero, una breve reflexión comparativa sobre el papel de las tres formas de aprendizaje cuantificadas por los indicadores del presente trabajo apunta en direcciones pertinentes: el aprendizaje mediante I+D a nivel interno tiene un papel superior a la absorción de informaciones y conocimientos externos capaces de generar innovaciones de impacto para el mercado nacional, en comparación con el "aprendizaje práctico" y el "aprendizaje mediante capacitación", en cualquiera de los casos (patrones) estudiados. Además, se destaca la aplicación de los conocimientos generados directamente por la I+D a las innovaciones (doble efecto) en todos los casos analizados.

Ya el hecho de que el papel del "aprendizaje práctico" haya demostrado su importancia para la absorción tecnológica, sea entre los sectores tecnológicamente más dinámicos (patrón 3) o entre los menos dinámicos (patrón 2), indica que los conocimientos obtenidos a través de las rutinas de manufactura permiten que las empresas tengan acceso a informaciones y conocimientos externos con diferentes niveles de complejidad.

El aprendizaje basado en la capacitación, por haberse mostrado tan solo a través del modelo 1 (causalidad indirecta), amplía la asimilación de conocimientos externos útiles para las innovaciones de producto de gran efecto, pero no parece tener repercusión directa en la generación de innovaciones.

Cabe destacar dos elementos que surgen de los resultados. En primer lugar, el hecho de "saber quién" tiene las informaciones y los conocimientos y "saber cómo" acceder a ellos es decisivo para el desarrollo de las innovaciones. Esos conocimientos se encuentran en el entorno externo a la empresa, pero también en el interno, y tener acceso a ellos no se deriva únicamente de las actividades de I+D, incluso en el caso de las innovaciones de gran repercusión. En relación con esto, se destaca que los objetivos de las políticas tecnológicas no pueden, por lo tanto, limitarse exclusivamente a estimular las actividades de I+D en las empresas.

\section{Bibliografía}

Albuquerque, E. (2000), "Domestic patents and developing countries: arguments for their study and data from Brazil (1980-1995)", Research Policy, vol. 29, $\mathrm{N}^{\circ}$ 9, Amsterdam, Elsevier.

Arbussa, A. y G. Coenders (2007), "Innovation activities, use of appropriation instruments and absorptive capacity: evidence from Spanish firms", Research Policy, vol. 36, $\mathrm{N}^{\circ} 10$, Amsterdam, Elsevier.

Arora, A. y A. Gambardella (1994), "Evaluating technological information and utilizing it: scientific knowledge, technological capability and external linkages in biotechnology", Journal of Economic Behavior and Organization, vol. 24, $\mathrm{N}^{\circ} 1$, Amsterdam, Elsevier.

Arrow, K.J. (1962), "The economic implications of learning by doing", Review of Economic Studies, vol. 29, № 3, Oxford, Oxford University Press.

Bell, M. y K. Pavitt (1995), "The development of technological capabilities", Trade, Technology and International Competitiveness, I.U. Haque y otros (eds.), Washington, D.C., Banco Mundial.

(1993), "Technological accumulation and industrial growth: contrast between developed and developing countries", Industrial and Corporate Change, vol. 2, $\mathrm{N}^{\circ}$ 2, Oxford, Oxford University Press.

Bittencourt, P.F. (2012), "Padrões setoriais de aprendizagem da indústria brasileira: uma análise exploratória", Revista Brasileira de Inovação, vol. 11, № 1, Campinas.

(2010), "Aprendizagem e absorção tecnológica na indústria brasileira: três ensaios sobre fatores da inovação na indústria brasileira”, tesis de doctorado, Río de Janeiro, Universidad Federal Fluminense.

Breschi, S. y F. Malerba (1997), "Sectoral innovation systems, technological regimes, Schumpeterian dynamics and spatial boundaries", Systems of Innovation: Technologies, Institutions and Organization, C. Edquist (eds.), Londres, Pinter.
Caloghirou, Y., I. Kastelli y A. Tsakanikas (2004), "Internal capabilities and external knowledge sources: complements or substitutes for innovative performance?", Technovation, vol. 24, Amsterdam, Elsevier.

Cassiman, B. y R. Veugelers (2006), "In search of complementarity in innovation strategy: internal R\&D and external knowledge acquisition”, Management Science, vol. 52, N ${ }^{\circ}$ 1, Institute for Operations Research and the Management Sciences.

Cassiolato, J.E. y H.M.M. Lastres (2003), "O foco em arranjos produtivos e inovativos locais de micro e pequenas empresas", Pequena empresa: cooperação e desenvolvimento local, H.M.M. Lastres, J.E. Cassiolato y M.L. Maciel, Río de Janeiro, Relume Dumará.

Chesbrough, H.W. y D.J. Teece (1996), "When is virtual virtuous? Organizing for innovation”, Harvard Business Review, vol. 74, $\mathrm{N}^{\circ}$ 1, Boston, Harvard Business Publishing.

Cohen, W.M., y D.A. Levinthal (1990), "Absorptive capacity: a new perspective of learning and innovation”, Administrative Science Quarterly, vol. 35, $\mathrm{N}^{\circ} 1$, Sage Publications.

(1989), "Innovation and learning: the two faces of R\&D", The Economic Journal, vol. 99, N ${ }^{\circ} 397$, St. Andrews, Royal Economic Society.

Cooke, P. y K. Morgan (1998), The Associational Economy: Firms, Regions, and Innovation, Nueva York, Oxford University Press.

De Negri, F. (2006), "Determinantes da inovação e da capacidade de absorção nas firmas brasileiras: qual o perfil da mão de obra?", documento presentado en el Encuentro de la Asociación Nacional de Centros de Posgrado en Economía (ANPEC).

Dosi, G. (1988), "The nature of innovative process", Technical Change an Economic Theory, G. Dosi y otros (eds.), Londres, Pinter.

Edquist, C. (2001), "The system of innovation approach and innovation policy: an account of the state of the art", Aalborg, Danish Research Unit for Industrial Dynamics, junio. 
Efron, B. y R. Tibshirani (1993), An Introduction to the Bootstrap, Boca Raton, Florida, Chapman \& Hall.

Figueiredo, P.N. (2004), "Aprendizagem tecnológica e inovação industrial em economias emergentes: uma breve contribuição para o desenho e implementação de estudos empíricos e estratégias no Brasil", Revista Brasileira de Inovação, vol. 3, $\mathrm{N}^{\circ}$ 2, Campinas, julio-diciembre.

Freeman, C. (1991), "Networks of innovators: a synthesis of research issues", Research Policy, vol. 20, $\mathrm{N}^{\circ}$ 5, Amsterdam, Elsevier. (1987), Technology, Policy and Economic Performance: Lessons from Japan, Londres, Printer.

Hedberg, B. (1981), "How organizational learn and unlearn?", Handbook of Organization Design, P.C. Nystrom y W.H. Starbuck (eds.), Londres, Oxford University Press.

Jensen, M.B. y otros (2007), "Forms of knowledge and modes of innovation", Research Policy, vol. 36, № 5, Amsterdam, Elsevier.

Kim, L.y R. Nelson (2005), "Introdução", Tecnologia, aprendizado e inovação: as experiências das economias de industrialização recente, Campinas, Editora Unicamp.

Laurensen, K. y A. Salter (2006), "Open for innovation: the role of openness in explaining innovative performance among UK manufacturing firms", Strategic Management Journal, vol. 27.

Legendre, P. y L. Legendre (1998), Numerical Ecology, Amsterdam, Elsevier.

Lundvall, B-Å. (2002), "The university in the learning economy", DRUID Working Paper, $\mathrm{N}^{\circ}$ 02-06, Aalborg, DRUID.

(org.) (1992), National Systems of Innovation: Towards a Theory of Innovation and Interactive Learning, Londres, Pinter.

Lundvall, B-Å y otros (2002), "National systems of production, innovation and competence building", Research Policy, vol. 31, $\mathrm{N}^{\circ}$ 2, Amsterdam, Elsevier.

Maillat, D. (1996), "Regional productive systems and innovative milieux", Network of Enterprises and Local Development, París, Organización de Cooperación y Desarrollo Económicos (OCDE).

Malerba, F. (2002), "Sectoral systems of innovation and production", Research Policy, vol. 31, $\mathrm{N}^{\circ}$ 2, Amsterdam, Elsevier.

(1992), "Learning by firms and incremental technical change", The Economic Journal, vol. 102, № 403, St. Andrews, Royal Economic Society.

Mowery, D.C., J.E. Oxley y B.S. Silverman (1996), "Strategic alliances and interfirm knowledge transfer", Strategic Management Journal, vol. 17.
Murovec, N. y I. Prodan (2009), "Absorptive capacity, its determinants, and influence on innovation output: cross-cultural validation of the structural model", Technovation, vol. $29, \mathrm{~N}^{\circ} 12$, Amsterdam, Elsevier.

Nelson, R.R. (1993), National Innovation Systems: a Comparative Analysis, Nueva York, Oxford University Press.

Nieto, M. y P. Quevedo (2005), "Absorptive capacity, technological opportunity, knowledge spillovers, and innovative effort", Technovation, vol. 25, $\mathrm{N}^{\circ} 10$, Amsterdam, Elsevier.

Pavitt, K. (1984), "Sectoral patterns of technical change: towards a taxonomy and a theory", Research Policy, vol. 13, $\mathrm{N}^{\circ} 6$, Amsterdam, Elsevier.

Pisano, G. (1996), "Learning-before-doing in the development of new process technology", Research Policy, vol. 25, $\mathrm{N}^{\circ} 7$, Amsterdam, Elsevier.

Schmidt, T. (2005), "What determines absorptive capacity", documento presentado en la DRUID Summer Conference 2005 on Dynamics of Industry and Innovation: Organizations, Networks and Systems, Copenhague.

Stock, G.N., N. Greis y W.A. Fischer (2001), "Absorptive capacity and new product development", Journal of High Technology, Management Research, vol. 12, $\mathrm{N}^{\circ}$ 1, Amsterdam, Elsevier.

Tsai, K.H. (2009), "Collaborative networks and product innovation performance: toward a contingency perspective", Research Policy, vol. 38, $\mathrm{N}^{\circ}$ 5, Amsterdam, Elsevier.

Tsai, K.H. y J. Wang (2009), "External technology sourcing and innovation performance in LMT sectors: an analysis based on the Taiwanese technological innovation survey", Research Policy, vol. 38, $\mathrm{N}^{\circ} 3$, Amsterdam, Elsevier.

Vega-Jurado, J.V. y otros (2008), "The effect of external and internal factors on firms' product innovation", Research Policy, vol. 37, $\mathrm{N}^{\circ} 4$, Amsterdam, Elsevier.

Veugelers, R. (1997), "Internal R\&D expenditures and external technology sourcing", Research Policy, vol. 26, $\mathrm{N}^{\circ} 3$, Amsterdam, Elsevier.

Viotti, E.B. (2002), "National learning systems - A new approach on technological change in late industrializing economies and evidences from the cases of Brazil and South Korea", Technological Forecasting and Social Change, vol. 69, $\mathrm{N}^{\circ} 7$, Amsterdam, Elsevier.

Zahra, S. y G. George (2002), “Absorptive capacity: a review, reconceptualization and extension", Academy of Management Review, vol. 27, $\mathrm{N}^{\circ}$ 2, Briarcliff Manor, Academy of Management. 